\title{
Improved reliability assessment of backup battery storage integrated with power supply system in a building
}

\author{
Muhammad Murtadha Othman ${ }^{1}$, Muhamad Amirul Naim Mohd Jamaluddin, Faisal Fauzi ${ }^{3}$, \\ Ismail Musirin ${ }^{4}$, Mohammad Lutfi Othman ${ }^{5}$ \\ ${ }_{1,2,3,4}$ Faculty of Electrical Engineering, Universiti Teknologi MARA, Malaysia \\ ${ }^{5}$ Centre for Advanced Power and Energy Research and Department of Electrical and Electronics Engineering, \\ Faculty of Engineering, Universiti Putra Malaysia, Malaysia
}

\begin{tabular}{l} 
Article Info \\
\hline Article history: \\
Received Aug 19, 2018 \\
Revised Feb 2, 2019 \\
Accepted Mar 24, 2019 \\
\hline Keywords: \\
Battery storage \\
Commercial power supply (CS) \\
Engine generator (EG) \\
Markov chain \\
Reliability
\end{tabular}

\section{Corresponding Author:}

Muhammad Murtadha Othman, Faculty of Electrical Engineering, Universiti Teknologi MARA, 40450 Shah Alam, Selangor, Malaysia.

Email:mamat505my@yahoo.com

\begin{abstract}
This paper presents the improved analysis of reliability for battery storage used in power system. The reliability assessment of this paper includes the evaluation of reliability of the system components, battery module and power electronic components. Battery storage is considered as one of energy storage and energy source that commonly used in power system. The evaluation of the reliability of power systems utilising with the storage batteries is performed by using the Markov chain process. The computation of the reliability is conducted by referring to the generated reliability block begins from power supply system. Every part of the system is evaluated regarding two specific states that are in normal or failure mode. By using the Markov method, the system unavailability and failure frequency can be computed.
\end{abstract}

Copyright (C) 2019 Institute of Advanced Engineering and Science. All rights reserved.

\section{INTRODUCTION}

Most of the electrical system of a building is becoming more advance in its operation due to its dependency on information and communication technology (ICT) that has increased in coming years. System failures caused by a single major harm originated from ICT will also instigate to a massive disaster to the society and economic of a country [1-6]. An electrical component operating having the criteria of high reliability is one of the reasons that it needs to be protected especially under the environment of ICT utilization. Hence, it is important to make sure that the communications buildings and data centers that operate ICT equipment needs to be stable, supplied by good power quality and without interruptions from the electrical supply system. Most of the buildings may have installed with ICT that have to be supplied by the power source without interruption. Usually, this type of building is operating with backup power sources such as uninterruptable power supplies (UPS). Therefore, a building having a backup power supply system needs a good planning that must be taken into consideration [7-12]. This can be achieved with the reliability assessment for every electrical component of battery storage, inverter, switch, commercial power supply and engine generator.

Hence, the Markov model is customarily used to analyze the reliability of a power supply integrated with the battery storage. This method is widely used in reliability analysis of repairable components. Reliability can be defined by a complex relationship of design, environment, test equipment, and maintenance skills amongst other influential factors [13-15]. There are numerous researches concerning with the utilization of Markov model for analyzing the reliability of power system and its components [16-18]. 
The continuous-time Markov chain is normally used in the reliability assessment of battery storage integrated with power supply system of a building. Reliability problems in power systems are usually occurred based on discrete in system and continuous in time. This is the reason that continuous-time Markov chain is suitable for reliability analysis of battery storage integrated with power supply. Electrical parts like generator, rectifier, inverter, and uninterruptable power supply (UPS) tend to have problems continuously in it states until a transition occurs which change the problem discretely to another state. A Markov chain is a mathematical system involving the transitions from one state to another according to certain probabilistic rules. The Markov chain is basically constructed based on the states of the Markov model. Every state consists of failure rate and repair rate and it is determined by observation in a specific time [13-15]. There are two types of configuration of reliability which are series and parallel systems. For series systems, it only can function properly if all of the components involved function properly. In contrast with the parallel systems, other components are still operating during which the other component is failing to operate. In other words, the whole system will not operate when there is failure for all of the components. Block diagram of power supply system for AC feeding as shown in Figure 1.

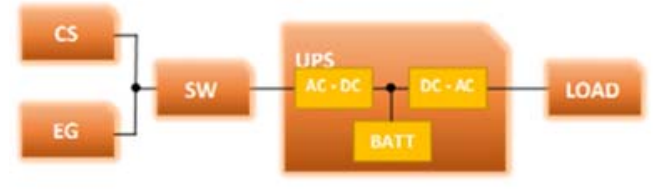

\author{
CS: Commercial Power Supply \\ EG: Engine-generator set \\ SW: Switchgear \\ UPS: Uninterruptable power supply \\ BATT: Battery
}

Figure 1. Block diagram of power supply system for AC feeding

An uninterruptible power supply (UPS) and standby generators are usually integrated with the power supply installed in a building [19-22]. In designing and planning the backup power supply system, it has to be installed with highly cost-effective investment. UPS system is widely used to make sure the power quality and sustainable power supplied to the electrical system of a building. It is possible to equip the UPS with long battery back-up time and small in its sizing. UPS systems with a longer lasting of battery back-up time can provide both quality and reliable power backup to the electrical system of a building [23-25]. A new method for calculating the unavailability Usys and failure frequency of power supply system with backup storage battery is introduced to achieve a precise result. By using the same power supply system configuration, the operation of the state transition is changed so that a better calculation can be made. In contrast to the basic approach, the new approach has the transition where the backup battery storage has to be recharged when exceeding the backup time before continue repairing the interrupted components. On the other hand, the new method gives an accurate value for the calculation of the unavailability Usys and failure frequency.

\title{
2. RESEARCH METHOD
}

This section will explain on the calculation of continuous-time Markov chain that used to determine the unavailability of a power system and also the reliability assessment of battery storage operating condition used at a specific backup time. The following subsection will describe thoroughly on the calculation of Markov chain required for reliability assessment of battery storage integrated with power supply system.

\subsection{Power supply system with backup battery storage}

In this section, the evaluation of reliability for a power supply system is introduced by applying the Markov process method which includes the reliability assessment of battery storage. Markov process is a method that uses the state transitions for failures for every component involved in order to create a Markov diagram and a set of probabilities that can be generated for every state transition the reliability evaluation. It is important to consider the backup time for the operation of battery storage. Figure 2 shows the configuration of power supply system with backup battery storage. From the configuration, failure mode for every component in the system is classified and represented into reliability blocks shown in Figure 3 . For the $\mathrm{CS} / \mathrm{EG}$ block, it represents the commercial power supply parallel with emergency engine generator. Then, block $\mathrm{A}, \mathrm{B}$ and $\mathrm{C}$ represent as the UPS components and the failures that affect each block are presented in Table 1. By referring Figure 3, CS/EG and block A, and also battery and block B is connected in series. The two branches are connected to a junction and is series with block $\mathrm{C}$.

Improved reliability assessment of backup battery storage integrated ... (Muhammad Murtadha Othman) 
Table 1. Components for each block of power supply system with backup battery storage

\begin{tabular}{cc}
\hline Blocks & Main Failure Mode \\
\hline CS / EG & Outage, Start-up failure \\
A & Thyristor (open), MCCB, Filter \\
B & Thyristor (short), Inverter \\
C & MCCB, Filter, Reactor \\
\hline
\end{tabular}

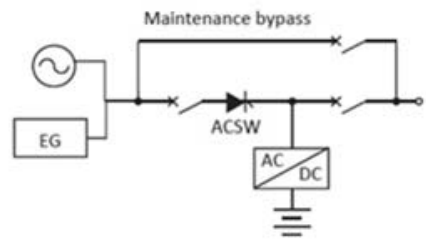

Figure 2. Power supply system configuration with backup storage battery

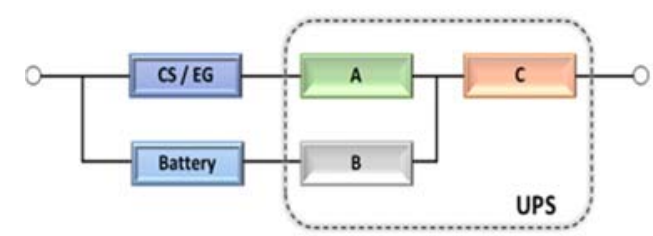

Figure 3. Reliability block diagram of power supply system with backup battery storage

\subsection{Reliability calculation for the power supply system with backup battery storage}

The reliability calculation for the power supply system with backup battery storage is comprised with the transition of failure for every block that falls under two conditions and this refers to FigError! Reference source not found.ure 3 . The first condition is the transition where the failure occurs at block a but there is still power supplied from the backup battery storage via block B shown in FigError! Reference source not found.ure 3. This first condition is represented by state transition P2 shown in FigError! Reference source not found.ure 4 . The second transition is where the failure happened at block A and battery storage exceeds the backup time shown in FigError! Reference source not found.ure 3. This state of transition falls under block P3 shown in FigError! Reference source not found.ure 4. For this type of interruption, the failure rate is derived from an equation that depends on the battery storage backup time as given in (1). Prior to the aforementioned two conditions, the state transition P1 represents as there is no interruption of power supply. In FigError! Reference source not found.ure 3, the transition from state P3 toP1 happened when block $\mathrm{A}$ is repaired without recharging the battery storage that exceeds the backup time as shown in FigError! Reference source not found.ure 3. In FigError! Reference source not found.ure 4, the repair rate of $\mu_{1}$ is set for the transition from P3 to P1. In particular, the transition from P2 to P3 is set to be $\alpha_{1}$ and from $\mathrm{P} 2$ to $\mathrm{P} 1$ is set to be $\beta_{1}$. On the other hand, the operation of state transition from P6 to P7 is relatively similar to the state transition from P2 to P3 shown in FigError! Reference source not found.ure 4. The only difference is that the failure is occurred at CS/EG block and there is still backup supply from battery storage that flows through block B shown in FigError! Reference source not found.ure 3. In FigError! Reference source not found.ure 4, the transition from state P6 to P7 is happened when the operation of battery is exceeding the backup time during the failure of at CS/EG block that refers to FigError! Reference source not found.ure 3. Therefore, the failure rate is referred as $\alpha_{2}$ for the state transmission from P6 to P7, and the state transition from P6 to P1 presented by $\beta_{2}$. In FigError! Reference source not found.ure 4, the transition from state P7 to P1 happened when CS/EG block is repaired without recharging the battery storage that exceeds the backup time as shown in FigError! Reference source not found.ure 3. In FigError! Reference source not found.ure 4, the repair rate of $\mu_{4}$ is set for the transition from P7 to P1. Therefore, the failure rate for state transition from P2 to P3, and also from P6 to P7 can be computed by using (2) and (3).

From (2) and (3) are derived from (1). The failure rate, $\lambda$ for all the state transitions is given as from the manual data. The repair rate of $\mu$ and $\beta$ for all the state transitions are calculated by using the equations which will be discussed in Section II.C. In other situation whereby CS/EG and block A is operating, while block B is in failure, hence the three-phase system is still receiving the electric supply from the CS/EG. This can be referred to Figure 3. Therefore, the state transition from P1 to P4 shows the failure of block B and vice-versa as depicted in Figure 4. In Figure 3, the failure of block $\mathrm{C}$ may cause to a complete interruption of electrical supply to the three-phase system. The failure of block $\mathrm{C}$ is represented by the state transition from P1 to P5 shown in Figure 4. By repairing block C, the CS/EG or backup battery storage will supply the power to the three-phase system depicted in Figure 3. Hence, by repairing the block $\mathrm{C}$, the state transition from $\mathrm{P} 5$ to P1 is incurred and it can be observed in Figure 4.

Int J Pow Elec \& Dri Syst, Vol. 10, No. 3, Sep 2019 : 1538 - 1546 


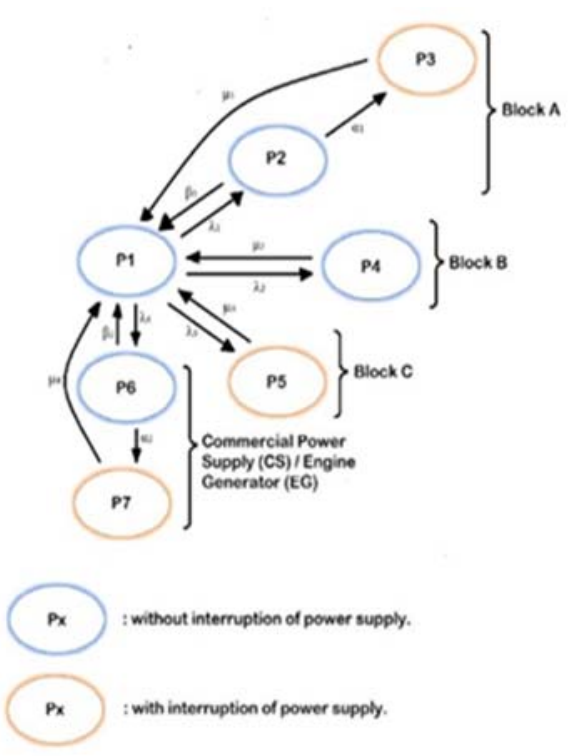

Figure 4. Markov chain diagram of power supply system with backup battery storage

The Markov chain diagram for the power supply system with backup battery storage given in Figure 4 is used to derive the transitional probability matrix given in (5). Then, the transitional probability matrix is used to calculate the probability of every state.

$$
\begin{aligned}
& \frac{P 3}{P 2}=\frac{\int_{T}^{\infty} \mu_{A} \cdot e^{(-\mu T)} d t}{\int_{0}^{T} \mu_{A} \cdot e^{(-\mu T)} d t}=\frac{e^{\left(-\mu_{A} T\right)}}{1-e^{\left(-\mu_{A} T\right)}} \\
& \alpha_{1}=\frac{\mu_{A} \cdot e^{\left(-\mu_{A} T\right)}}{1-e^{\left(-\mu_{A} T\right)}} \\
& \alpha_{2}=\frac{\mu_{C S / E G} \cdot e^{\left(-\mu_{\left.C S / E G^{T}\right)}\right.}}{1-e^{\left(-\mu_{C S / E G^{T)}}\right.}} \\
& \beta=\mu
\end{aligned}
$$

The failure frequency for the system can be referred to the state transition to a total failure that is from $\mathrm{P} 2$ to $\mathrm{P} 3, \mathrm{P} 1$ to $\mathrm{P} 5$, and $\mathrm{P} 6$ to $\mathrm{P} 7$.

\subsection{Estimation of repair rate, $\mu$}

Table 2 represents the failure rate $\lambda$ for every state transition shown in Figure 4 . The repair rate for state transition is estimated based on the mean time to repair (MTTR) given in equation (6). MTTR is defined as the average time needed to repair any components that are failed to operate and return back to normal operating conditions [7].

$$
\mu=\frac{1}{M T T R}(\text { repair } / \text { hour })
$$

The MTTR for CS/EG is assumed to be 10 hours for every repair condition, and for block A, B and $\mathrm{C}$ are assumed to be 5 hours for every repair condition. The MTTR for battery is estimated to be 2 hours of repair condition. Therefore, the repair rate for each block can be calculated by using the following equations.

$$
\left.\mu_{C S / E G}=\frac{1}{10}=0.1 \text { (repair } / \text { hour }\right)
$$

Improved reliability assessment of backup battery storage integrated ... (Muhammad Murtadha Othman) 


$$
\begin{aligned}
& \mu_{A, B, C}=\frac{1}{5}=0.2(\text { repair } / \text { hour }) \\
& \mu_{B a t t e r y}=\frac{1}{2}=0.5(\text { repair } / \text { hour })
\end{aligned}
$$

Table 2. Failure rate $\lambda$ for every state transition

\begin{tabular}{cc}
\hline Blocks & Failure rate $\lambda$ \\
\hline CS / EG & $2.5 \times 10^{-3}$ \\
A & $7 \times 10^{-7}$ \\
B & $3 \times 10^{-5}$ \\
C & $9 \times 10^{-7}$ \\
\hline
\end{tabular}

The obtained repair rates $\mu$ and failure rates $\lambda$ will be used in the state transition matrix of Markov chain in (5) to calculate the probabilities of every state.

\subsection{Improved reliability assessment of power supply system with backup battery storage}

This section illustrates a new approach used to design the Markov chain diagram that represents the changes in the operation of power supply system with backup battery storage. Therefore, changes of Markov chain diagram from Figure 4 can be seen in Figure 6. By referring to Figure 4, the only changes made are referring to the state transition from $\mathrm{P} 3$ to $\mathrm{P} 1$, and also from $\mathrm{P} 7$ to $\mathrm{P} 1$. This means that the state transition is changed from P3 to P2 and also from P7 to P6 shown in Figure 6. In Figure 6, the state transition from P3 to $\mathrm{P} 2$ resemblance during the failure of block A and battery, the battery is given enough time to recharge and then it is connected to the system once the components in block A is repaired and this referring to Figure 3. Therefore, the new state transition between P2 and P3 can be observed in Figure 5 and Figure 6.

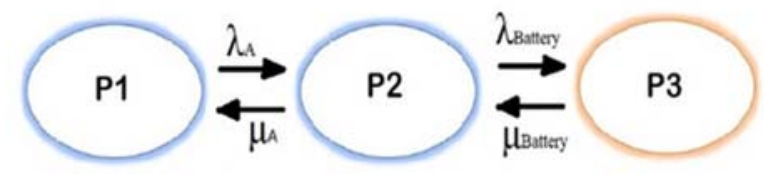

Figure 5. Markov chain for the new state transition between P2 and P3

By referring to the Markov chain diagram shown in Figure 5, the stochastic transitional probability matrix for the new state transition between P2 and P3 is given in (10).

$$
P=\left[\begin{array}{ccc}
1-\lambda_{A} & \lambda_{A} & 0 \\
\mu_{A} & 1-\left(\mu_{A}+\lambda_{\text {Battery }}\right) & \lambda_{\text {Battery }} \\
0 & \mu_{\text {Battery }} & 1-\mu_{\text {Battery }}
\end{array}\right]
$$

The new state probability P1, P2 and P3 given in (11), (12) and (13) are derived from (10).

$$
\begin{aligned}
& P_{1}=\frac{\mu_{A}}{\left(\lambda_{A}+\mu_{A}\right)} \\
& P_{2}=\frac{\lambda_{A} \mu_{\text {Battery }}}{\left(\lambda_{A}+\mu_{A}\right)\left(\lambda_{\text {Batter } \left.y^{+} \mu_{\text {Battery }}\right)}\right.} \\
& P_{3}=\frac{\lambda_{\text {Battery }}}{\lambda_{\text {Battery }}+\mu_{\text {Battery }}}
\end{aligned}
$$

From (14), (15) and (16) are derived directly from the Markov chain diagram given in Figure 5.

$$
\lambda_{A} P 1=\mu_{A} P 2
$$




$$
\begin{aligned}
& \left(\lambda_{\text {Battery }}+\mu_{A}\right) P 2=\lambda_{A} P 1+\mu_{\text {Battery }} P 3 \\
& \mu_{\text {Battery }} P 3=\lambda_{\text {Battery }} P 2
\end{aligned}
$$

Solve (10) to determine the battery failure rate, $\lambda_{\text {Battery, }}$, which depends on the backup time given in (17). From (17) is basically originated from (1).

$$
\lambda_{\text {Battery }}=\mu_{\text {Battery }} \cdot \frac{P 3}{P 2}=\frac{\mu_{\text {Battery }} \cdot e^{\left(-\mu_{\text {Battery }} T\right)}}{1-e^{\left(-\mu_{B a t t e r y}\right)}}
$$

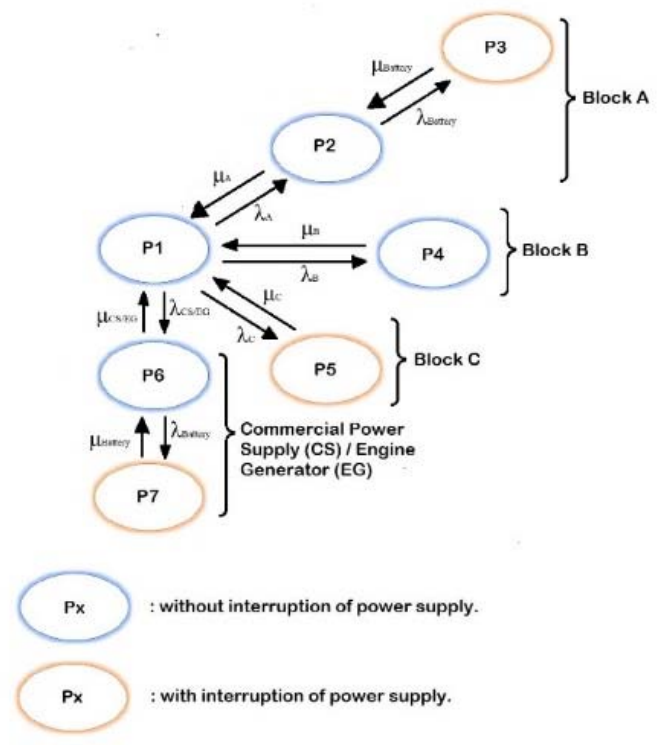

Figure 6. A new Markov diagram for power supply system with backup battery storage

It is worth to mention that the state transition is also changed from P7 to P6 shown in Figure 6. In Figure 6, the state transition from $\mathrm{P} 7$ to $\mathrm{P} 6$ shows during the failure of $\mathrm{CS} / \mathrm{EG}$ and battery, the battery is given enough time to recharge and then it is connected back to the system once the CS/EG is repaired and this referring to Figure 6.

The stochastic transitional probability matrix for the for the new Markov chain of power supply system with backup battery storage is given in (18).

$$
P=\left[\begin{array}{ccccccc}
1-\left(\lambda_{A}+\lambda_{B}+\lambda_{C}+\lambda_{C S / E G}\right) & \lambda_{A} & 0 & \lambda_{B} & \lambda_{C} & \lambda_{C S / E G} & 0 \\
\mu_{A} & 1-\left(\mu_{A}+\lambda_{\text {Battery }}\right) & \lambda_{\text {Battery }} & 0 & 0 & 0 & 0 \\
0 & \mu_{\text {Battery }} & 1-\mu_{\text {Battery }} & 0 & 0 & 0 & 0 \\
\mu_{B} & 0 & 0 & 1-\mu_{B} & 0 & 0 & 0 \\
\mu_{C} & 0 & 0 & 0 & 1-\mu_{C} & 0 & 0 \\
\mu_{C S / E G} & 0 & 0 & 0 & 0 & 1-\left(\mu_{C S / E G}+\lambda_{\text {Battery }}\right) & \lambda_{\text {Battery }} \\
0 & 0 & 0 & 0 & 0 & \mu_{\text {Battery }} & 1-\mu_{\text {Battery }}
\end{array}\right]
$$

The probability for every state is used to calculate the risk of the system and is given in (19) until (25).

$$
\begin{aligned}
& P_{1}=\frac{\mu_{A} \mu_{B} \mu_{C} \mu_{C S / E G}}{\left(\lambda_{A}+\mu_{A}\right)\left(\lambda_{B}+\mu_{B}\right)\left(\lambda_{C}+\mu_{C}\right)\left(\lambda_{C S / E G}+\mu_{C S / E G}\right)} \\
& P_{2}=\frac{\lambda_{A} \mu_{\text {Battery }}}{\left(\lambda_{A}+\mu_{A}\right)\left(\lambda_{\text {Battery }}+\mu_{\text {Battery })}\right.}
\end{aligned}
$$




$$
\begin{aligned}
& P_{3}=\frac{\lambda_{\text {Battery }}}{\lambda_{\text {Battery }}+\mu_{\text {Battery }}} \\
& P_{4}=\frac{\lambda_{B}}{\left(\lambda_{B}+\mu_{B}\right)} \\
& P_{5}=\frac{\lambda_{C}}{\left(\lambda_{C}+\mu_{C}\right)} \\
& P_{6}=\frac{\lambda_{C S / E G} \mu_{\text {Battery }}}{\left(\lambda_{C S / E G}+\mu_{C S / E G}\right)\left(\lambda_{\text {Batter } y}+\mu_{\text {Battery }}\right)} \\
& P_{7}=\frac{\lambda_{\text {Battery }}}{\lambda_{\text {Battery }}+\mu_{\text {Battery }}}
\end{aligned}
$$

The unavailability of the three-phase system, $U_{s y s}$, is the sum of the probabilities of state facing complete failure. The complete failure is happened when there is no power supply because of the battery storage exceeding the backup time during the failure of $\mathrm{CS} / \mathrm{EG}$, block A or block $\mathrm{C}$ that refers to Figure 3. For this situation, the $U_{s y s}$ calculated by referring to the summation of P3, P5 and P7 representing as a complete failure for the three-phase system given in (26).

$$
U_{\text {sys }}=P 3+P 5+P 7
$$

The failure frequency for the system can be referred to the state transition to a total failure that is from $\mathrm{P} 2$ to $\mathrm{P} 3, \mathrm{P} 1$ to $\mathrm{P} 5$, and $\mathrm{P} 6$ to $\mathrm{P} 7$ given in (27).

$$
f_{f}=f_{f 1}+f_{f 2}+f_{f 6}=P 1\left(\lambda_{C}\right)+P 2\left(\lambda_{\text {Battery }}\right)+P 6\left(\lambda_{\text {Battery }}\right)
$$

\section{RESULTS AND ANALYSIS}

This section will discuss on the reliability assessment based on the unavailability and failure frequency for both of the basic and improved Markov chain of power supply system with the backup battery storage. The probability for each state of the basic Markov chain and the new Markov chain of power supply with the backup battery storage and the calculated value is tabulated in Table 3 . The total probability of basic approach of power supply with the backup battery storage is obtained in a large value that is $7.55 \times 10^{10}$ and this concept is incorrect since the value should be limited to a unity value. Therefore, an improved version of Markov chain for power supply with the backup battery storage is introduced and is shown in Figure 6.

Table 3. Probability for every state of basic and improved Markov chain for power supply with the backup battery storage

\begin{tabular}{ccc}
\hline State & $\begin{array}{c}\text { Probability of the basic method } \\
\text { (p.u.) }\end{array}$ & $\begin{array}{c}\text { Probability of the improved } \\
\text { method (p.u.) }\end{array}$ \\
\hline P1 & 0.975 & 0.975 \\
P2 & $7.545 \times 10^{10}$ & $1.4 \times 10^{-6}$ \\
P3 & 5 & $8.194 \times 10^{-40}$ \\
P4 & $1.5 \times 10^{-4}$ & $1.5 \times 10^{-4}$ \\
P5 & $4.5 \times 10^{-6}$ & $4.5 \times 10^{-6}$ \\
P6 & $1.601 \times 10^{7}$ & 0.024 \\
P7 & 10 & $8.194 \times 10^{-40}$ \\
\hline
\end{tabular}

By using (26) that refers to the basic approach of power supply with the backup battery storage, the calculated unavailability for the operating condition of three-phase system is 15 and this is referring to the battery backup time of 3 hours. By using (26) that refers to the improved approach of power supply with the backup battery storage, the unavailability for the operating condition of three-phase system is $4.5 \times 10^{-6}$ and this is also referring to the battery backup time of 3 hours. The variation of $U_{s y s}$ at every time interval for both basic and improved approach of power supply with the backup battery storage can be observed via Figure 7 and Figure 8 .

Int J Pow Elec \& Dri Syst, Vol. 10, No. 3, Sep 2019 : $1538-1546$ 


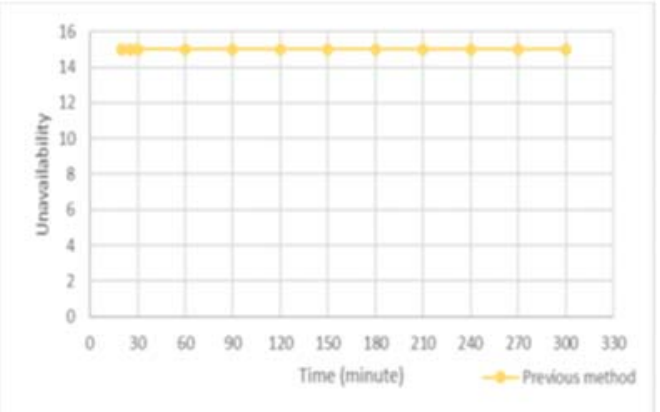

Figure 7. Variation of $U_{\text {sys }}$ at every time interval for the basic approach of power supply with the backup battery storage

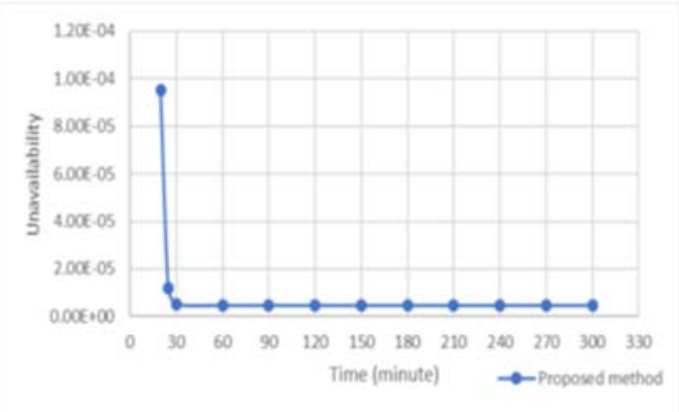

Figure 8. Variation of $U_{\text {sys }}$ at every time interval for the improved approach of power supply with the backup battery storage

The results signify that the improved approach of power supply with the backup battery storage provides a persuasively accurate result of $U_{s y s}$ in contrast with the peculiarities large value in the result of $U_{s y s}$ obtained based on the basic approach of power supply with the backup battery storage. The failure frequency of $2.439 \times 10^{-2}$ and $8.779 \times 10^{-7}$ for the basic and improved concept of power supply with the backup battery storage are obtained, respectively. The failure frequency value of $8.779 \times 10^{-7}$ is chosen because it is obtained based on the accurate estimation of total state probability and $U_{s y s}$ determined by referring to the improved approach of power supply with the backup battery storage.

\section{CONCLUSION}

This paper has presented an improved method of Markov chain in evaluating the reliability of power supply system with backup battery storage. The unavailability and the failure frequency have been evaluated by implementing the continuous-time Markov chain for both basic and improved approach of power supply with the backup battery storage. The calculated unavailability for the new Markov chain of power supply system with backup battery storage is $4.5 \times 10^{-6}$. Unlike the basic method, the new method presents that it is improved to get an accurate result for the unavailability of the system. The improved method also gives a better result of failure frequency with computed value of $8.779 \times 10^{-7}$. The changes of the operating transition where the battery has to be charged after exceeding the backup time resulting to a better reliability assessment of the power supply system with backup battery storage. Cognizance towards the importance of unavailability and failure frequency for the power supply system with backup battery storage will render to a high reliability three-phase system.

\section{ACKNOWLEDGEMENTS}

This research was supported by the Long-Term Research Grant (LRGS), Ministry of Education Malaysia for the program titled "Decarbonisation of Grid with an Optimal Controller and Energy Management for Energy Storage System in Microgrid Applications" with project code 600-IRMI/LRGS 5/3 $(001 / 2019)$. The authors would also like to acknowledge The Institute of Research Management \& Innovation (IRMI), Universiti Teknologi MARA (UiTM), Shah Alam, Selangor, Malaysia for the facilities provided to support on this research.

\section{REFERENCES}

[1] T. Tsumura, K. Hirose, and M. Mino, "Application of a Markov Process to Evaluate the Reliability of the Power Supply Systems Utilizing Storage Batteries,” 35th Int. Telecommun. Energy Conf. 'Smart Power Effic, 2013.

[2] I. A. Tøndel, J. Foros, S. S. Kilskar, P. Hokstad, and M. G. Jaatun, "Interdependencies and reliability in the combined ICT and power system: An overview of current research," Applied computing and informatics, vol. 14, no. 1, pp. 17-27, 2018.

[3] S. C. Müller, H. Georg, J. J. Nutaro, E. Widl, Y. Deng, P. Palensky, M. U. Awais, M. Chenine, M. Küch, M. Stifter, and H. Lin, "Interfacing power system and ict simulators: Challenges, state-of-the-art, and case studies," IEEE Transactions on Smart Grid, vol. 9, no. 1, pp. 14-24, 2018. 
[4] D. Schacht, S. Patzack, H. Vennegeerts, S. Krahl, and A. Moser, "Selection of relevant failure modes and system states for the evaluation of reliability in distribution grids depending on ICT," CIRED Workshop 2016, pp. 9-4, 2016.

[5] D. Schacht, D. Lehmann, H. Vennegeerts, S. Krahl, and A. Moser, "Modelling of interactions between power system and communication systems for the evaluation of reliability," In 2016 Power Systems Computation Conference (PSCC), pp. 1-7, 2016.

[6] H. Lei, and C. Singh, "Developing a benchmark test system for electric power grid cyber-physical reliability studies," In IEEE 2016 International conference on probabilistic methods applied to power systems (PMAPS), pp. $1-5,2016$.

[7] T. Tsumura, T. Takeda, and K. Hirose, "A Tool for Calculating Reliability of Power Supply for Information and Communication Technology Systems," INTELEC'08, pp. 1-6, 2008.

[8] P. Palensky, and D. Dietrich, "Demand side management: Demand response, intelligent energy systems, and smart loads," IEEE Transactions on Industrial Informatics, vol. 7, no. 3, pp. 381-388, 2011.

[9] H. C. Gils, Y. Scholz, T. Pregger, D. L. de Tena, and D. Heide, "Integrated modelling of variable renewable energy-based power supply in Europe," Energy, vol. 123, pp. 173-188, 2017.

[10] X. Luo, J. Wang, M. Dooner, and J. Clarke, "Overview of current development in electrical energy storage technologies and the application potential in power system operation," Applied energy, vol. 137, pp. 511-536, 2015.

[11] M. Mohammadi, R. Ghasempour, F. R. Astaraei, E. Ahmadi, A. Aligholian, and A. Toopshekan, "Optimal planning of renewable energy resource for a residential house considering economic and reliability criteria," International Journal of Electrical Power \& Energy Systems, vol. 96, pp. 261-273, 2018.

[12] S. Weitemeyer, D. Kleinhans, L. Siemer, and C. Agert, "Optimal combination of energy storages for prospective power supply systems based on Renewable Energy Sources," Journal of Energy Storage, vol. 20, pp. 581-589, 2018.

[13] F. Wayne, "Philosophy of Engineering Reliability," pp. 579-583, 1832.

[14] S. Rao M, V.N.A Naikan, "Availability modeling of repairable systems using Markov system dynamics simulation," International Journal of Quality \& Reliability Management, vol. 32, no. 5, pp. 517-531, 2015.

[15] W. Bamrungsetthapong, and A. Pongpullponsak, "Parameter interval estimation of system reliability for repairable multistate series-parallel system with fuzzy data," The Scientific World Journal, vol. 2014, 2014.

[16] R. H. Zaini, M. M. Othman, I. Musirin, A. Mohamed, and A. Hussain. "Determination of transmission reliability margin considering uncertainties of system operating condition and transmission line outage." European Transactions on Electrical Power, vol. 21, no. 1, pp. 380-397, 2011.

[17] N. A. Salim, M. M. Othman, M. S. Serwan, M. Fotuhi-Firuzabad, A. Safdarian, and I. Musirin, "Determination of available transfer capability with implication of cascading collapse uncertainty," IET Generation, Transmission \& Distribution, vol. 8, no. 4, pp. 705-715, 2014.

[18] M. M. Othman, N. Abd Rahman, I. Musirin, M. Fotuhi-Firuzabad, and A. Rajabi-Ghahnavieh, "A heuristic ranking approach on capacity benefit margin determination using Pareto-based evolutionary programming technique," The Scientific World Journal, vol. 2015, 2015.

[19] T. Tsumura, H. Murai, K. Hirose, and M. Yamasaki, "Development of a Calculating System Using Power Supply Reliability Field Data in Japan," 10th Int. Conf. Electr. Power Qual. Util., 2009.

[20] A. K. Shukla, K. Sudhakar, and P. Baredar, "A comprehensive review on design of building integrated photovoltaic system," Energy and Buildings, vol. 128, pp. 99-110, 2016.

[21] N. Arshad, and U. Ali, "An analysis of the effects of residential uninterpretable power supply systems on Pakistan's power sector," Energy for Sustainable Development, vol. 36, pp. 16-21, 2017.

[22] M. Aamir, K. A. Kalwar, and S. Mekhilef, "Uninterruptible power supply (ups) system," Renewable and Sustainable Energy Reviews, vol. 58, pp. 1395-1410, 2016.

[23] Q. Han, "Data-telecom Power System Solution Based on Reliability Analysis," 29th Int. Telecommun. Energy Conf. (INTELEC '07), pp. 917-922, 2007.

[24] M. Aamir, K. A. Kalwar, and S. Mekhilef. "Uninterruptible power supply (ups) system." Renewable and Sustainable Energy Reviews, vol. 58, pp. 1395-1410, 2016.

[25] X. Luo, J. Wang, M. Dooner, and J. Clarke, "Overview of current development in electrical energy storage technologies and the application potential in power system operation," Applied energy, vol. 137, pp. 511-536, 2015.

Int J Pow Elec \& Dri Syst, Vol. 10, No. 3, Sep 2019 : 1538 - 1546 